\title{
Determination of neuron-specific enolase in patients with midgut-type tumour treated with somatostatin analogues
}

\author{
Paweł Gut ${ }^{(1)}$, Agata Czarnywojtek ${ }^{(12}$, Nadia Sawicka-Gutaj ${ }^{(1)}$, Kosma Woliński ${ }^{\circledR 1}$, \\ Adam Maciejewski ${ }^{\circledR 1}$, Paweł Komarnicki', Marek Ruchała ${ }^{(01}$ \\ ${ }^{1}$ Department of Endocrinology, Metabolism, and Internal Diseases, Poznan University of Medical Sciences, Poznan, Poland \\ ${ }^{2}$ Department of Pharmacology, Poznan University of Medical Sciences, Poznan, Poland
}

\begin{abstract}
Introduction: The biochemical diagnosis of neuroendocrine tumours (NETs) uses assays of specific and nonspecific markers. Nonspecific markers include, among others, neuron-specific enolase (NSE). The aim of this study was to evaluate NSE in patients with midgut type tumours treated with somatostatin analogues.

Material and methods: The study group of patients with NETs of the small intestine included 41 patients. Grade G1 was found in 19 cases, while G2 was seen in the remaining 22 cases. Liver metastases were found in all patients studied. The examined group of patients was treated with somatostatin analogues receiving octreotide LAR at a dose of $30 \mathrm{mg}$. The control of biochemical parameters was performed every 3 months and imaging examinations every 6 months. The Immuno-Biological Laboratories kit was used for determination of NSE concentration, where reference values were $12.5-25 \mathrm{ng} / \mathrm{mL}$.

Results: In the G1 group of patients, the median value of NSE concentration was $134.67 \mathrm{ng} / \mathrm{mL}$, while in the G2 group, the value was 234.55 $\mathrm{ng} / \mathrm{mL}$ and was significantly higher than in the G1 group $(\mathrm{p}=0.003)$. In the determination of NSE concentration values according to the degree of liver involvement, in the group of patients with $10 \%$ liver involvement, the median value of NSE concentration was $143.21 \mathrm{ng} / \mathrm{mL}$, while in the group with $25 \%$ liver involvement, the value was $251.82 \mathrm{ng} / \mathrm{mL}(\mathrm{p}<0.001)$. In the analysis of NSE concentration assessment in patients with disease progression, the median value was $234.65 \mathrm{ng} / \mathrm{mL}$ compared to the group with disease stabilization, where the median NSE value was significantly lower and amounted to $136.27 \mathrm{ng} / \mathrm{mL}(\mathrm{p}<0.001)$.

Conclusions: In our study, we observed that NSE concentration values were significantly higher among patients with NET midgut type tumour with histological grade G2 and in patients with $25 \%$ liver involvement and progression of the disease process. (Endokrynol Pol 2021; 72 (4): 308-318)
\end{abstract}

Key words: neuron-specific enolase; neuroendocrine tumours; midgut; somatostatin analogues

\section{Introduction}

The assessment of the endocrine function of gastrointestinal neuroendocrine tumours (NETs) is an important step in the diagnosis and treatment monitoring of these diseases. Specific and non-specific markers of neuroendocrine tumours are used in biochemical diagnostics [1-3]. Non-specific markers include chromogranin A (CgA), neuron-specific enolase (NSE), and the $\alpha$ and $\beta$ subunits of human chorionic gonadotropin (hCG). NSE has lower sensitivity and specificity in the diagnosis of NETs than CgA [4,5]. NSE levels are elevated in 50-70\% of patients with carcinoid tumour, pancreatic islet tumour, pheochromocytoma, medullary thyroid cancer, and small cell lung cancer $[6,7]$. Physiologically, NSE occurs in the central and peripheral nervous system, pituitary, adrenal medulla, and pineal gland. Its elevated values may also be found in septic shock and post-traumatic states. The simultaneous determination of CgA and NSE demon- strates higher sensitivity than of each of these markers separately [8-10]. Neuron-specific enolase is a useful marker in the diagnosis of low and highly differentiated tumours. NSE concentrations roughly correlate with tumour mass and disease stage. Simultaneous determination of $\mathrm{CgA}$, pancreatic polypeptide (PP), and NSE may increase the sensitivity in the diagnosis of NETs, especially endocrine inactive pancreatic NETs and carcinoid tumour [11-12]. Landry et al. present in their work the dependence of NSE levels on tumour size, presence of metastasis to surrounding and distant lymph nodes, histological maturity, and presence of vascular infiltration [13]. The study by Adrichem et al. shows that NSE is a general biomarker of survival in patients with clinical stage IV of NET according to the TNM classification [14]. High NSE values indicate a more aggressive disease course and faster progression. The aim of this study was to evaluate NSE in patients with midgut type tumour treated with somatostatin analogues. 


\section{Material and methods}

All examined patients who underwent surgical removal of the primary focus with histopathological evaluation according to the $2017 \mathrm{WHO}$ classification. All patients underwent thorough diagnostic imaging (abdominal ultrasonography, CT scan of chest, abdomen, and pelvis) and complementary biochemical tests like CgA, serotonin, 5-hydroxyindoleacetic acid (5-HIAA), and NSE to assess clinical staging. Receptor scintigraphy with ${ }^{99 m}$ Tc-EDDA/ /HYNIC-TOC was performed in each case to qualify to treatment with somatostatin analogues. The intensity of radioactive tracer accumulation in liver metastases was evaluated using the qualitative scale developed by E.P. Krenning (grade 0-4) [15]. In the group of patients studied, the degree of radioactive tracer accumulation in the liver was in grades 3 and 4 according to the Krenning scale. The examined group of patients was treated with somatostatin analogues from 2015 to 2019, receiving octreotide LAR at a dose of $30 \mathrm{mg}$ every 4 weeks. The control of biochemical parameters was performed every 3 months. Imaging examinations were performed every 6 months in order to obtain an objective assessment of the response to treatment using RECIST 1.1 criteria. For the determination of NSE levels, a kit from Immuno-Biological Laboratories (Minneapolis, Minnesota, USA) was used, where reference values ranged from 12.5 to $25 \mathrm{ng} / \mathrm{mL}$.

\section{Statistical evaluation}

The calculations were made using Statistica 13 by TIBCO and PQStat by PQStat Software. The level of significance was $\alpha=0.05$. The result was considered statistically significant when $\mathrm{p}<\alpha$. The normality of the distribution of variables was tested with the Shapiro-Wilk test. The Wilcoxon-Mann-Whitney-U test was used to compare the variables between the two groups. In order to test whether the changes over time of NSE are statistically significant because of not conforming to the normal distribution, the Friedman ANOVA test was used with the Dunn-Bonferroni multiple comparison test.

\section{Results}

The study group of patients diagnosed with NET of the small intestine consisted of 41 patients, including 29 women $(70.7 \%)$ and 12 men $(29.3 \%)$. The mean age of males was $60.41 \pm 4.90$ years while that of females was $64.20 \pm 10.39$ years. Maturity grade G1 was found in 19 (46.3\%) tissue specimens, while G2 (53.7\%) was found in the remaining 22 specimens. Liver metastases were found in all patients ( $10 \%$ liver involvement in 23 cases, $25 \%$ liver involvement in 18 cases) (Tab. 1 ). The aim of our study was to assess the concentration of NSE in patients treated with somatostatin analogues. NSE determinations were performed every 3 months. The study was conducted in the period 2015-2019, obtaining 16 NSE determinations for analysis in each patient, which are presented in the attached tables. The results of the study were presented as a comparison of groups of patients depending on the degree of liver involvement, grading, or stage of disease. The changes in the analysed parameters over time were also assessed.

\section{Evaluation of the results of NSE concentration values in relation to grading}

In the group of patients with histological tumour maturity grade G1 $(n=19)$, the median value of NSE concentration was $134.67 \mathrm{ng} / \mathrm{mL}$, while in the group with histological maturity grade $\mathrm{G} 2(\mathrm{n}=22)$, this value was $234.55 \mathrm{ng} / \mathrm{mL}$, which was significantly higher than in the G1 group $(p=0.003)$. An analogous relationship was observed in the analysis of the median of the last NSE concentration values, i.e. in the G1 group the median of last NSE values was $199.67 \mathrm{ng} / \mathrm{mL}$ and in the G2 group it was $538.05 \mathrm{ng} / \mathrm{mL}$ ( $\mathrm{p}=0.001$ ) (Tab. 2, 3). The assessment of changes in NSE during therapy showed statistically significant differences in the G1 and G2 groups. In both groups there was an increase in NSE values, but it was significantly faster in the G2 group (Tab. 8, 9) and (Fig. 1-3).

\section{Evaluation of the results of NSE concentration values in relation to the degree of liver involvement}

In the analysis of NSE concentration values according to the degree of liver involvement, the median value of NSE concentration in the group of patients with $10 \%$ liver involvement $(\mathrm{n}=23)$ was $143.21 \mathrm{ng} / \mathrm{mL}$, while in the group with $25 \%$ liver involvement $(n=18)$ this value was significantly higher and amounted to $251.82 \mathrm{ng} / \mathrm{mL}$ $(p<0.001)$. In the analysis of the last NSE concentration values in the first group, the median of the last values was $221.34 \mathrm{ng} / \mathrm{mL}$, which was significantly lower compared to the second group, in which NSE concentration was $570.73 \mathrm{ng} / \mathrm{mL}(\mathrm{p}<0.001)$ (Tab. 4, 5). Analysis of the variables over time showed that the NSE values in patients with liver involvement in $25 \%$ increased during treatment with somatostatin analogues much faster and by a higher order of value (Tab. 10, 11) (Fig. 4-6).

\section{Evaluation of NSE concentration results in relation to disease stage}

In the analysis of NSE concentration, the median value in the group of patients with disease progression $(\mathrm{n}=21)$ was $234.65 \mathrm{ng} / \mathrm{mL}$ compared to the group with disease stabilization $(n=20)$, where the median value of NSE concentration was significantly lower and equal to 136.27 $\mathrm{ng} / \mathrm{mL}(\mathrm{p}<0.001)$. On the other hand, in the analysis of the last values, the median NSE concentration in the group with disease progression (PD) was $543.12 \mathrm{ng} / \mathrm{mL}$, which was significantly higher than the group with disease stabilization (SD), in which the NSE concentration value was $210.45 \mathrm{ng} / \mathrm{mL}(\mathrm{p}<0.001$ ) (Tab. 6, 7). The assessment of NSE values in patients with disease stabilization performed during treatment showed a tendency for a slow increase in the value, but much lower than in the group of patients with disease progression (Tab. 12, 13) (Fig. 7-9). 
Table 1. Characteristics of patients with midgut neuroendocrine tumours

\begin{tabular}{|c|c|c|c|c|c|c|c|}
\hline Number & Patient ID & Gender (F/M) & Age (years) & $\begin{array}{l}\text { Primary } \\
\text { tumour }\end{array}$ & $\begin{array}{l}\text { Liver involvement } \\
\text { by metastasis (\%) }\end{array}$ & Ki-67 (\%) & Grading (G1/G2) \\
\hline 1. & 2015-01 & $\mathrm{F}$ & 60 & Ileum & 10 & 2 & G1 \\
\hline 2. & 2015-02 & M & 65 & Ileum & 10 & 2 & G1 \\
\hline 3. & 2015-03 & $\mathrm{F}$ & 75 & Ileum & 25 & 5 & G2 \\
\hline 4. & 2015-04 & $\mathrm{F}$ & 53 & Jejunum & 25 & 10 & G2 \\
\hline 5. & 2015-05 & $\mathrm{F}$ & 67 & Ileum & 10 & 10 & G2 \\
\hline 6. & 2015-06 & $M$ & 60 & Jejunum & 10 & 4 & G2 \\
\hline 7. & 2015-07 & $\mathrm{F}$ & 73 & Jejunum & 25 & 5 & G2 \\
\hline 8. & 2015-08 & $\mathrm{F}$ & 70 & Ileum & 10 & 10 & G2 \\
\hline 9. & 2015-09 & $\mathrm{F}$ & 67 & Jejunum & 10 & 5 & G2 \\
\hline 10. & 2015-10 & $\mathrm{F}$ & 78 & Jejunum & 25 & 10 & G2 \\
\hline 11. & 2015-11 & $\mathrm{F}$ & 55 & Ileum & 10 & 1 & G1 \\
\hline 12. & 2015-12 & M & 60 & Ileum & 10 & 4 & G2 \\
\hline 13. & 2015-13 & $\mathrm{F}$ & 69 & Ileum & 25 & 5 & G2 \\
\hline 14. & 2015-14 & $\mathrm{F}$ & 49 & Ileum & 25 & 10 & G2 \\
\hline 15. & 2015-15 & $\mathrm{F}$ & 73 & Jejunum & 25 & 10 & G2 \\
\hline 16. & 2015-16 & $\mathrm{F}$ & 33 & Jejunum & 10 & 4 & G2 \\
\hline 17. & 2015-17 & $\mathrm{F}$ & 58 & Jejunum & 10 & 2 & G1 \\
\hline 18. & 2015-18 & M & 61 & Ileum & 10 & 2 & G1 \\
\hline 19. & 2015-19 & M & 65 & Ileum & 10 & 2 & G1 \\
\hline 20. & 2015-20 & M & 66 & Jejunum & 25 & 5 & G2 \\
\hline 21. & $2015-21$ & $\mathrm{~F}$ & 71 & Ileum & 25 & 2 & G1 \\
\hline 22. & 2015-22 & $\mathrm{F}$ & 68 & Ileum & 10 & 2 & G1 \\
\hline 23. & 2015-23 & $\mathrm{F}$ & 80 & Ileum & 10 & 2 & G1 \\
\hline 24. & 2015-24 & $\mathrm{F}$ & 67 & Ileum & 10 & 2 & G1 \\
\hline 25. & 2015-25 & M & 63 & Jejunum & 10 & 2 & G1 \\
\hline 26. & 2015-26 & $\mathrm{F}$ & 65 & Ileum & 25 & 10 & G2 \\
\hline 27. & 2015-27 & $\mathrm{F}$ & 71 & Ileum & 10 & 1 & G1 \\
\hline 28. & 2015-28 & M & 66 & Jejunum & 25 & 10 & G2 \\
\hline 29. & $2015-29$ & $\mathrm{~F}$ & 67 & Jejunum & 10 & 2 & G1 \\
\hline 30. & 2015-30 & $M$ & 51 & Ileum & 10 & 2 & G1 \\
\hline 31. & 2015-31 & $\mathrm{F}$ & 77 & Jejunum & 25 & 10 & G2 \\
\hline 32. & 2015-32 & $\mathrm{F}$ & 48 & Jejunum & 10 & 2 & G1 \\
\hline 33. & 2015-33 & $\mathrm{M}$ & 54 & Ileum & 25 & 5 & $\mathrm{G} 2$ \\
\hline 34. & 2015-34 & $\mathrm{F}$ & 65 & Jejunum & 10 & 2 & G1 \\
\hline 35. & 2015-35 & $\mathrm{F}$ & 67 & Ileum & 25 & 5 & G2 \\
\hline 36. & 2015-36 & $\mathrm{F}$ & 59 & Jejunum & 25 & 5 & $\mathrm{G} 2$ \\
\hline 37. & 2015-37 & $\mathrm{F}$ & 51 & Ileum & 25 & 10 & G2 \\
\hline 38. & 2015-38 & M & 57 & Jejunum & 25 & 10 & G2 \\
\hline 39. & 2015-39 & $\mathrm{F}$ & 61 & Ileum & 10 & 2 & G1 \\
\hline 40. & 2015-40 & $M$ & 57 & Jejunum & 25 & 10 & G2 \\
\hline 41. & 2015-41 & $\mathrm{F}$ & 65 & Ileum & 10 & 2 & G1 \\
\hline
\end{tabular}


Table 2. Median of neuron-specific enolase (NSE) value depending on grading

\begin{tabular}{|c|c|c|c|c|c|c|c|c|c|}
\hline \multirow{2}{*}{ Grading } & \multicolumn{8}{|c|}{ NSE value [ng/mL] } & \multirow{2}{*}{$\mathbf{p}^{*}$} \\
\hline & $\mathbf{N}$ & Mean & SD & Median & Min & Max & 01 & 03 & \\
\hline G1 & 19 & 124.22 & 61.65 & 134.67 & 19.76 & 229.76 & 79.45 & 167.54 & 0.003 \\
\hline G2 & 22 & 254.40 & 93.35 & 234.55 & 62.34 & 458.32 & 187.45 & 324.12 & \\
\hline
\end{tabular}

*Wilcoxon-Mann-Whitney-U test; SD — standard deviation

Table 3. Median of last neuron-specific enolase (NSE) value depending on grading

\begin{tabular}{|c|c|c|c|c|c|c|c|c|c|}
\hline \multirow{2}{*}{ Grading } & \multicolumn{8}{|c|}{ Last NSE value [ng/mL] } & \multirow{2}{*}{$\mathbf{p}^{*}$} \\
\hline & $\mathbf{N}$ & Mean & SD & Median & Min & Max & 01 & 03 & \\
\hline G1 & 19 & 210.41 & 104.53 & 199.67 & 30.13 & 436.78 & 127.76 & 265.45 & 0.001 \\
\hline G2 & 22 & 483.22 & 161.09 & 538.05 & 87.98 & 673.45 & 352.12 & 621.12 & \\
\hline
\end{tabular}

*Wilcoxon-Mann-Whitney-U test; SD — standard deviation

Table 4. Median of neuron-specific enolase (NSE) value depending on the degree of liver involvement

\begin{tabular}{|c|c|c|c|c|c|c|c|c|c|}
\hline \multirow{2}{*}{$\begin{array}{l}\text { Liver involvement } \\
\text { degree }\end{array}$} & \multicolumn{8}{|c|}{ NSE value [ng/mL] } & \multirow{2}{*}{$\mathbf{p}^{*}$} \\
\hline & $\mathbf{N}$ & Mean & SD & Median & Min & Max & 01 & 03 & \\
\hline $10 \%$ & 23 & 134.69 & 61.38 & 143.21 & 19.76 & 229.76 & 86.55 & 180.54 & $<0.001$ \\
\hline $25 \%$ & 18 & 269.95 & 95.96 & 251.82 & 62.34 & 458.32 & 210.87 & 324.12 & \\
\hline
\end{tabular}

*Wilcoxon-Mann-Whitney-U test; SD — standard deviation

Table 5. Median of last neuron-specific enolase (NSE) value depending on the degree of liver involvement

\begin{tabular}{|c|c|c|c|c|c|c|c|c|c|}
\hline \multirow{2}{*}{$\begin{array}{l}\text { Liver involvement } \\
\text { degree }\end{array}$} & \multicolumn{8}{|c|}{ Last NSE value [ng/mL] } & \multirow{2}{*}{$\mathbf{p}^{*}$} \\
\hline & $\mathbf{N}$ & Mean & SD & Median & Min & Max & 01 & 03 & \\
\hline $10 \%$ & 23 & 223.91 & 98.87 & 221.34 & 30.13 & 426.67 & 153.21 & 278.76 & $<0.001$ \\
\hline $25 \%$ & 18 & 526.60 & 145.55 & 570.73 & 87.98 & 673.45 & 456.23 & 623.21 & \\
\hline
\end{tabular}

*Wilcoxon-Mann-Whitney-U test; SD — standard deviation

Table 6. Median of neuron-specific enolase (NSE) value depending on the stage of the disease

\begin{tabular}{|c|c|c|c|c|c|c|c|c|c|}
\hline \multirow{2}{*}{ Stage of disease } & \multicolumn{8}{|c|}{ NSE value [ng/mL] } & \multirow{2}{*}{$\mathbf{p}^{*}$} \\
\hline & $\mathbf{N}$ & Mean & SD & Median & Min & Max & 01 & $\mathbf{0 3}$ & \\
\hline PD & 21 & 259.07 & 93.28 & 234.65 & 62.34 & 458.32 & 210.87 & 324.12 & $<0.001$ \\
\hline SD & 20 & 125.83 & 59.95 & 136.27 & 19.76 & 229.76 & 83.00 & 172.94 & \\
\hline
\end{tabular}

*Wilcoxon-Mann-Whitney-U test; PD — progressive disease; SD — stable disease; SD — standard deviation

Table 7. Median of last neuron-specific enolase (NSE) value depending on the stage of the disease

\begin{tabular}{|c|c|c|c|c|c|c|c|c|c|}
\hline \multirow{2}{*}{ Stage of disease } & \multicolumn{8}{|c|}{ Last NSE value [ng/mL] } & \multirow{2}{*}{$\mathbf{p}^{*}$} \\
\hline & $\mathbf{N}$ & Mean & SD & Median & Min & Max & 01 & 03 & \\
\hline PD & 21 & 499.92 & 151.79 & 543.12 & 87.98 & 673.45 & 421.34 & 621.12 & $<0.001$ \\
\hline SD & 20 & 206.51 & 91.11 & 210.45 & 30.13 & 426.67 & 140.48 & 270.84 & \\
\hline
\end{tabular}

*Wilcoxon-Mann-Whitney-U test; PD — progressive disease; SD — stable disease; SD — standard deviation 
Table 8. Neuron-specific enolase (NSE) concentrations in patients with grading G1

\section{The number of NSE determinations at intervals of 3 months}

\begin{tabular}{lcccc} 
& Mean rank & Total rank & Mean & SD \\
\hline NSE 1 & 1.00000 & 19.0000 & 57.7147 & 41.5644 \\
\hline NSE 2 & 2.42105 & 46.0000 & 74.1505 & 48.1867 \\
\hline NSE 3 & 2.84211 & 54.0000 & 80.3674 & 46.2400 \\
\hline NSE 4 & 3.84211 & 73.0000 & 87.4858 & 47.1748 \\
\hline NSE 5 & 5.00000 & 95.0000 & 96.7147 & 50.5468 \\
\hline NSE 6 & 6.18421 & 117.5000 & 107.7821 & 54.4278 \\
\hline NSE 7 & 6.89474 & 131.0000 & 113.7668 & 57.5935 \\
\hline NSE 8 & 7.81579 & 148.5000 & 124.2274 & 64.6521 \\
\hline NSE 9 & 9.05263 & 172.0000 & 133.2142 & 68.9360 \\
\hline NSE 10 & 189.0000 & 143.3568 & 76.8494 \\
\hline NSE 11 & 9.94737 & 154.8663 & 81.8665 \\
\hline NSE 12 & 11.05263 & 164.3074 & 88.4108 \\
\hline NSE 13 & 12.05263 & 229.0000 & 178.8311 & 92.4878 \\
\hline NSE 14 & 12.94737 & 246.0000 & 190.0758 & 98.4470 \\
\hline NSE 15 & 14.05263 & 267.0000 & 202.0116 & 104.5348 \\
\hline NSE 16 & 15.05263 & 286.0000 & 210.4174 &
\end{tabular}
$\chi^{2}$ test, ANOVA $(\mathrm{N}=19, \mathrm{df}=15)=282.1391 \mathrm{p}=0.00000$ Kendall's compliance factor $=\mathbf{0 . 9 8 9 9 6}$ Group of patients with grading $\mathbf{G 1}$

ANOVA Friedman Test and Kendall's compliance factor

SD — standard deviation

Table 9. Neuron-specific enolase (NSE) concentration in patients with grading G2

The number of NSE determinations at intervals of 3 months
ANOVA Friedman Test and Kendall's compliance factor $\chi^{2}$ tes, ANOVA $(\mathrm{N}=22, \mathrm{df}=15)=325.7767 \mathrm{p}=0.00000$ Kendall's compliance factor $=0.98720$ Group of patients with grading $\mathbf{G 2}$

\begin{tabular}{|c|c|c|c|c|}
\hline & Mean rank & Total rank & Mean & SD \\
\hline NSE 1 & 1.04545 & 23.0000 & 104.9009 & 72.7653 \\
\hline NSE 2 & 1.95455 & 43.0000 & 120.6795 & 73.9144 \\
\hline NSE 3 & 3.00000 & 66.0000 & 139.5950 & 74.1617 \\
\hline NSE 4 & 4.04545 & 89.0000 & 165.4005 & 82.9654 \\
\hline NSE 5 & 5.04545 & 111.0000 & 185.1473 & 87.1361 \\
\hline NSE 6 & 6.04545 & 133.0000 & 208.9973 & 89.2591 \\
\hline NSE 7 & 6.86364 & 151.0000 & 233.1536 & 95.2373 \\
\hline NSE 8 & 8.04545 & 177.0000 & 254.4064 & 93.3510 \\
\hline NSE 9 & 9.04545 & 199.0000 & 282.9327 & 97.5109 \\
\hline NSE 10 & 10.13636 & 223.0000 & 311.7505 & 104.1570 \\
\hline NSE 11 & 11.27273 & 248.0000 & 334.4173 & 110.6949 \\
\hline NSE 12 & 11.86364 & 261.0000 & 355.7800 & 122.4481 \\
\hline NSE 13 & 13.00000 & 286.0000 & 387.9255 & 135.0418 \\
\hline NSE 14 & 13.90909 & 306.0000 & 414.4964 & 144.1943 \\
\hline NSE 15 & 14.81818 & 326.0000 & 454.1450 & 155.7836 \\
\hline NSE 16 & 15.90909 & 350.0000 & 483.2236 & 161.0939 \\
\hline
\end{tabular}




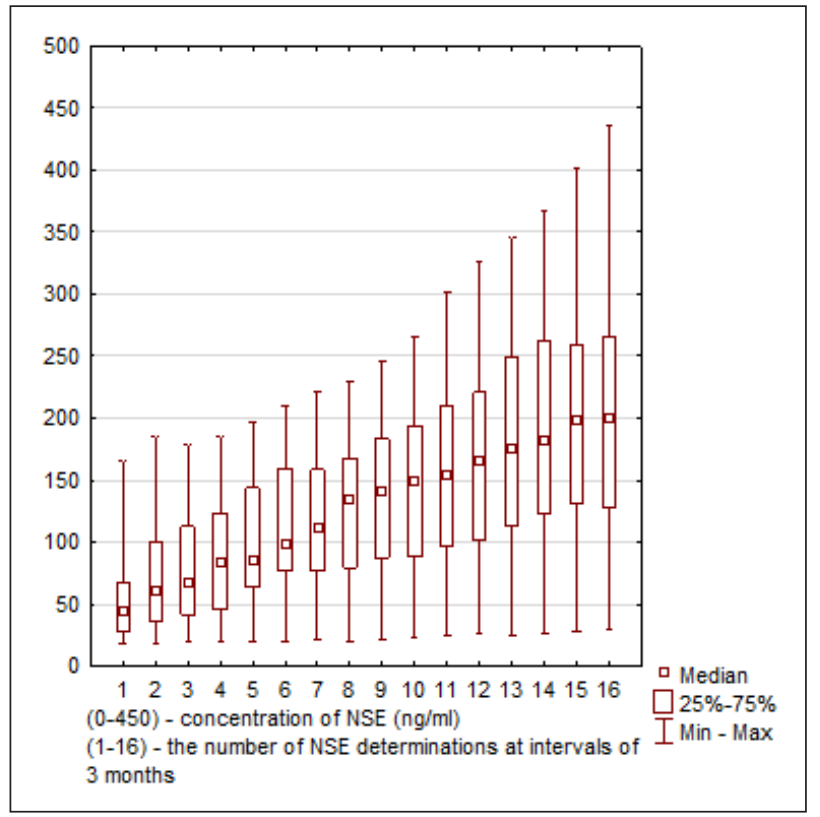

Figure 1. Neuron-specific enolase (NSE) concentrations in patients with G1 grading

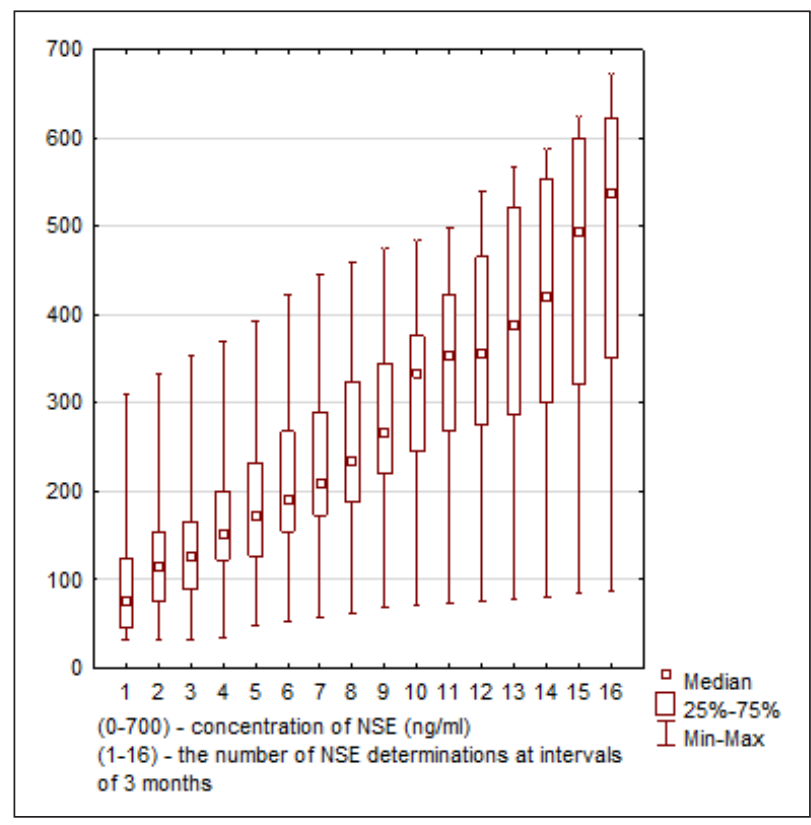

Figure 2. Neuron-specific enolase (NSE) concentrations in patients with $\mathrm{G} 2$ grading

\section{Discussion and Conclusions}

In our study, we observed that NSE concentration values were significantly higher among patients with histological maturity of neuroendocrine tumour grade G2 and in patients with $25 \%$ liver involvement and progression of the disease process. NSE may be elevated in $38-45 \%$ of low-grade NETs, and it is one of the important prognostic factors. We also noticed that

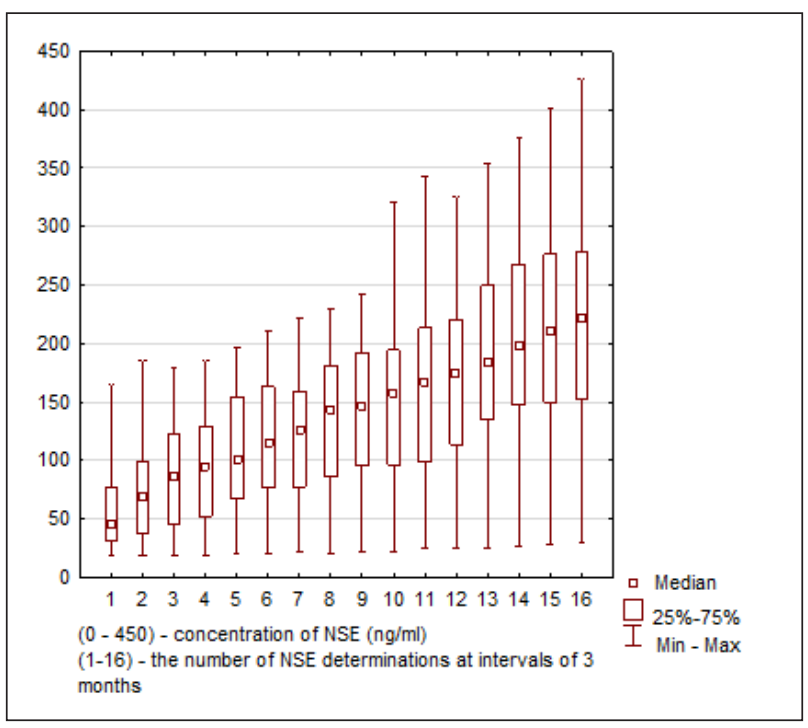

Figure 3. Neuron-specific enolase (NSE) concentrations in patients with G1 and G2 grading

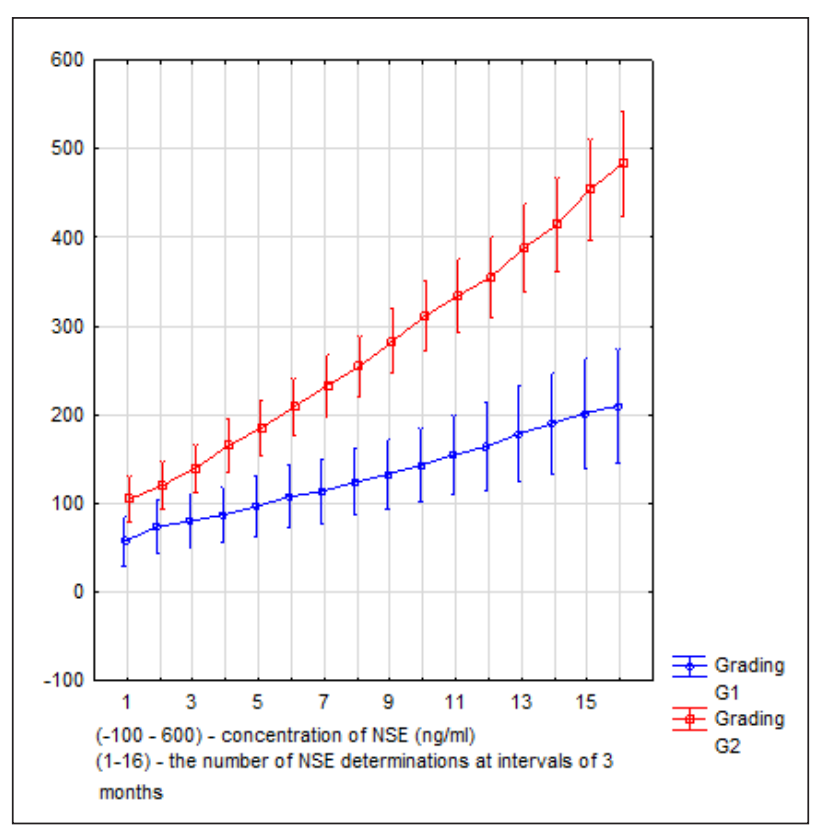

Figure 4. Neuron-specific enolase (NSE) concentrations in patients with $10 \%$ liver involvement

in all groups of patients undergoing treatment with somatostatin analogues an increase in NSE values was seen. It is worth noting that the increase in NSE was statistically greater in patients with disease progression, 25\% liver involvement, or G2 grading. The level of NSE correlates with tumour differentiation, aggressiveness, and tumour size and is inversely related to general survival and progression-free survival [16]. It is currently believed that the NSE level depends on the presence of metastases, their location, number, and size and correlates with response to treatment with 


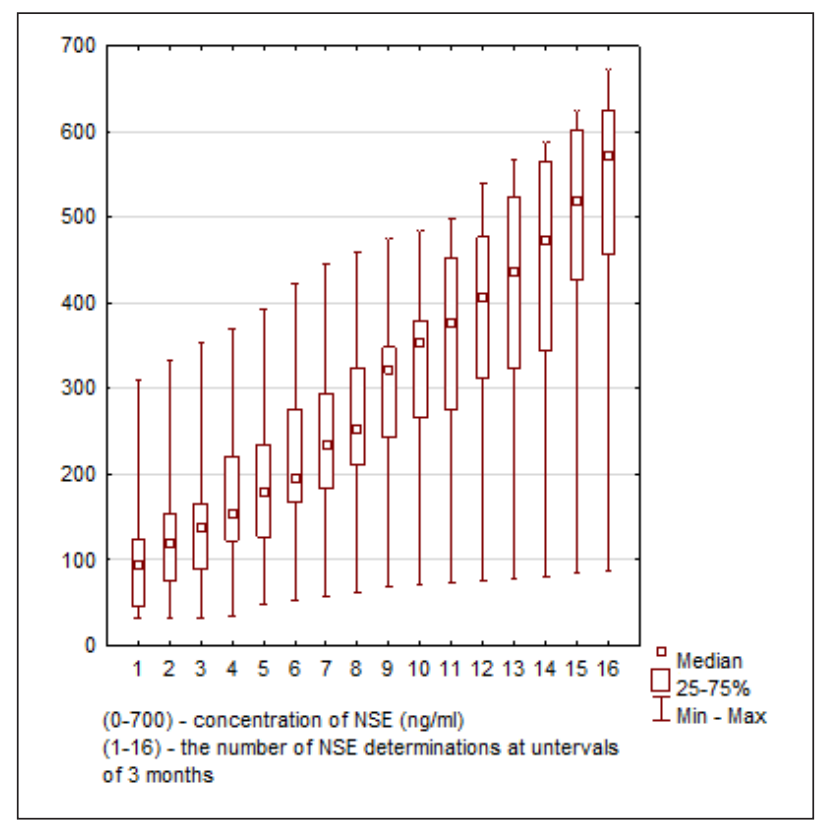

Figure 5. Neuron-specific enolase (NSE) concentrations in patients with $25 \%$ liver involvement

somatostatin analogues [17]. The authors of many reports show that the values of NSE in patients with neuroendocrine neoplasms tend to increase during treatment $[18,19]$. The rate of increase in concentration or the time to double the value depends on the

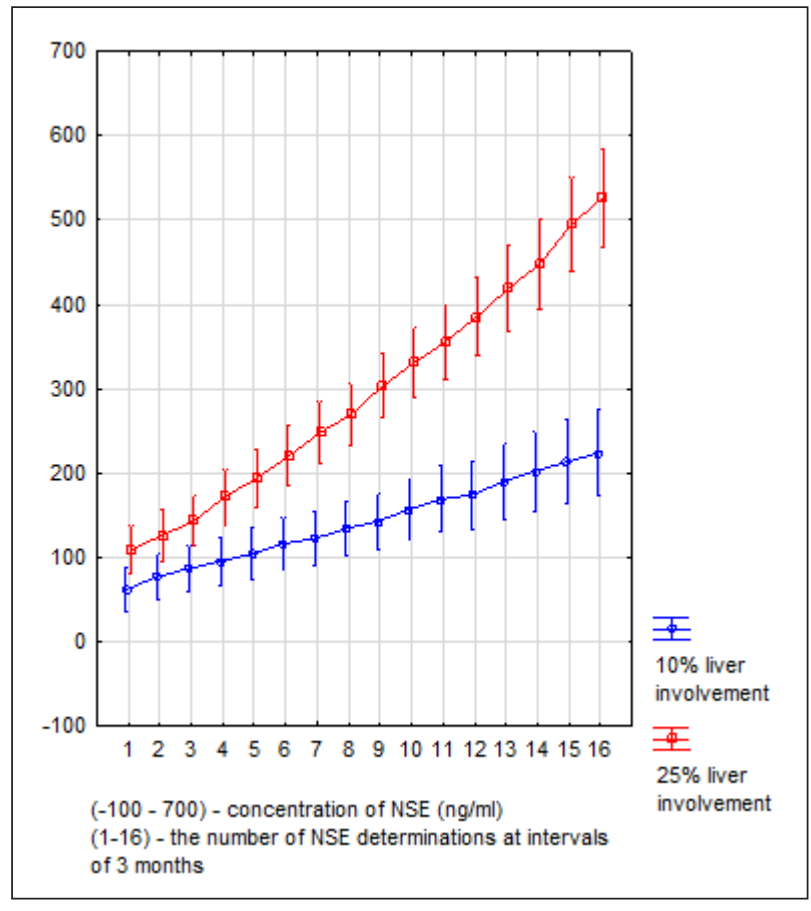

Figure 6. Neuron-specific enolase (NSE) concentrations in patients with $10 \%$ and $25 \%$ liver involvement

degree of malignancy and the course of the disease. It has been proven that faster NSE increases occur in patients with G2 or G3 grading and with high liver

Table 10. Neuron-specific enolase (NSE) concentrations in patients with $10 \%$ liver involvement

\begin{tabular}{|c|c|c|c|c|}
\hline \multirow[t]{2}{*}{$\begin{array}{l}\text { The number of NSE } \\
\text { determinations at intervals } \\
\text { of } 3 \text { months }\end{array}$} & \multicolumn{4}{|c|}{$\begin{array}{l}\text { ANOVA Friedman Test and Kendall's compliance factor } \\
\chi^{2} \text { test, ANOVA }(\mathrm{N}=23, \mathrm{df}=15)=337.9561 \mathrm{p}=0.00000 \\
\text { Kendalls' compliance factor }=0.97958 \\
\text { Group of patients with } 10 \% \text { liver involvement }\end{array}$} \\
\hline & Mean rank & Total rank & Mean & SD \\
\hline NSE 1 & 1.00000 & 23.0000 & 62.3422 & 43.27264 \\
\hline NSE 2 & 2.34783 & 54.0000 & 77.6848 & 47.15279 \\
\hline NSE 3 & 2.86957 & 66.0000 & 87.4813 & 46.70559 \\
\hline NSE 4 & 3.91304 & 90.0000 & 95.7813 & 48.60537 \\
\hline NSE 5 & 5.04348 & 116.0000 & 104.8413 & 51.00891 \\
\hline NSE 6 & 6.19565 & 142.5000 & 116.5817 & 54.36883 \\
\hline NSE 7 & 6.78261 & 156.0000 & 122.8222 & 57.09912 \\
\hline NSE 8 & 7.89130 & 181.5000 & 134.6974 & 61.38782 \\
\hline NSE 9 & 9.08696 & 209.0000 & 143.1761 & 63.00154 \\
\hline NSE 10 & 10.08696 & 232.0000 & 157.4835 & 75.31124 \\
\hline NSE 11 & 11.30435 & 260.0000 & 169.3274 & 81.67059 \\
\hline NSE 12 & 11.91304 & 274.0000 & 174.3604 & 78.56607 \\
\hline NSE 13 & 12.95652 & 298.0000 & 190.8513 & 85.52316 \\
\hline NSE 14 & 13.95652 & 321.0000 & 202.7343 & 89.72118 \\
\hline NSE 15 & 14.86957 & 342.0000 & 213.7426 & 93.84824 \\
\hline NSE 16 & 15.78261 & 363.0000 & 223.9122 & 98.87586 \\
\hline
\end{tabular}

SD — standard deviation 
Table 11. Neuron-specific enolase (NSE) concentrations in patients with $25 \%$ liver involvement

\section{The number of NSE determinations at intervals of 3 months}

\begin{tabular}{lcccc} 
& Mean rank & Total rank & Mean & SD \\
\hline NSE 1 & 1.05556 & 19.0000 & 109.4739 & 77.2166 \\
\hline NSE 2 & 1.94444 & 35.0000 & 126.5033 & 78.8214 \\
\hline NSE 3 & 3.00000 & 54.0000 & 143.6667 & 80.3969 \\
\hline NSE 4 & 4.00000 & 72.0000 & 172.1150 & 89.3396 \\
\hline NSE 5 & 5.00000 & 90.0000 & 194.4150 & 92.9599 \\
\hline NSE 6 & 6.00000 & 108.0000 & 220.2456 & 94.4134 \\
\hline NSE 7 & 7.00000 & 126.0000 & 248.1133 & 98.5946 \\
\hline NSE 8 & 8.00000 & 144.0000 & 269.9567 & 95.9628 \\
\hline NSE 9 & 9.00000 & 162.0000 & 303.4744 & 95.7778 \\
\hline NSE 10 & 10.00000 & 180.0000 & 331.1206 & 100.9483 \\
\hline NSE 11 & 11.00000 & 355.8394 & 106.9080 \\
\hline NSE 12 & 12.00000 & 216.0000 & 385.4839 & 114.6341 \\
\hline NSE 13 & 13.00000 & 234.0000 & 419.0317 & 128.9639 \\
\hline NSE 14 & 14.00000 & 252.0000 & 448.1928 & 136.8991 \\
\hline NSE 15 & 15.00000 & 270.0000 & 495.1850 & 141.1335 \\
\hline NSE 16 & 16.00000 & 288.0000 & 526.6039 & 145.5583
\end{tabular}

SD — standard deviation

Table 12. Neuron-specific enolase (NSE) concentrations in patients with stable disease (SD)

\section{The number of NSE} determinations at intervals of 3 months
ANOVA Friedman Test and Kendall's compliance factor $\chi^{2}$ test, ANOVA $(\mathrm{N}=18, \mathrm{df}=15)=269.9167 \mathrm{p}=0.00000$ Kendall's compliance factor $=0.99969$

Group of patients with $25 \%$ liver involvement

\begin{tabular}{|c|c|c|c|c|}
\hline & \\
\hline & Mean rank & Total rank & Mean & SD \\
\hline NSE 1 & 1.00000 & 20.0000 & 55.9090 & 39.20011 \\
\hline NSE 2 & 2.40000 & 48.0000 & 71.5840 & 45.69266 \\
\hline NSE 3 & 2.85000 & 57.0000 & 80.6815 & 44.60210 \\
\hline NSE 4 & 3.90000 & 78.0000 & 88.3710 & 46.70542 \\
\hline NSE 5 & 5.05000 & 101.0000 & 96.5590 & 48.72696 \\
\hline NSE 6 & 6.22500 & 124.5000 & 107.5600 & 51.89274 \\
\hline NSE 7 & 6.75000 & 135.0000 & 113.3280 & 54.22080 \\
\hline NSE 8 & 7.87500 & 157.5000 & 125.8365 & 59.95579 \\
\hline NSE 9 & 9.10000 & 182.0000 & 134.8905 & 62.35193 \\
\hline NSE 10 & 9.85000 & 197.0000 & 143.9380 & 66.76366 \\
\hline NSE 11 & 11.10000 & 222.0000 & 154.4945 & 71.91771 \\
\hline NSE 12 & 12.00000 & 240.0000 & 161.8815 & 73.80538 \\
\hline NSE 13 & 13.05000 & 261.0000 & 176.8930 & 81.19302 \\
\hline NSE 14 & 14.05000 & 281.0000 & 186.4670 & 83.16072 \\
\hline NSE 15 & 14.95000 & 299.0000 & 196.7600 & 86.16215 \\
\hline NSE 16 & 15.85000 & 317.0000 & 206.5180 & 91.11139 \\
\hline
\end{tabular}

ANOVA Friedman Test and Kendall's compliance factor $\chi^{2}$ test, ANOVA ( $\left.\mathrm{N}=20, \mathrm{df}=15\right)=295.9596 \mathrm{p}=0.00000$ Kendall's compliance factor $=\mathbf{0 . 9 8 6 5 3}$ Group of patients with SD

SD - standard deviation 
Table 13. Neuron-specific enolase (NSE) concentrations in patients with progressive disease (PD)

\begin{tabular}{|c|c|c|c|c|}
\hline \multirow[t]{2}{*}{$\begin{array}{l}\text { Number of NSE } \\
\text { determinations at intervals } \\
\text { of } 3 \text { months }\end{array}$} & \multicolumn{4}{|c|}{$\begin{array}{c}\text { ANOVA Friedman Test and Kendall's compliance factor } \\
\chi^{2} \text { test, ANOVA }(\mathrm{N}=21, \mathrm{df}=15)=311.9748 \mathrm{p}=0.00000 \\
\text { Kendall's compliance factor }=0.99040 \\
\text { Group of patients with PD }\end{array}$} \\
\hline & Mean rank & Total rank & Mean & SD \\
\hline NSE 1 & 1.04762 & 22.0000 & 108.8676 & 73.1644 \\
\hline NSE 2 & 1.95238 & 41.0000 & 125.3395 & 73.9349 \\
\hline NSE 3 & 3.00000 & 63.0000 & 142.1162 & 75.2625 \\
\hline NSE 4 & 4.00000 & 84.0000 & 168.2676 & 83.5643 \\
\hline NSE 5 & 5.00000 & 105.0000 & 189.5067 & 87.0499 \\
\hline NSE 6 & 6.00000 & 126.0000 & 214.0286 & 88.8238 \\
\hline NSE 7 & 7.00000 & 147.0000 & 239.2567 & 94.1239 \\
\hline NSE 8 & 8.00000 & 168.0000 & 259.0729 & 93.2857 \\
\hline NSE 9 & 9.00000 & 189.0000 & 288.4657 & 96.7623 \\
\hline NSE 10 & 10.23810 & 215.0000 & 319.2157 & 100.7601 \\
\hline NSE 11 & 11.23810 & 236.0000 & 343.3214 & 106.9528 \\
\hline NSE 12 & 11.90476 & 250.0000 & 367.2081 & 117.0264 \\
\hline NSE 13 & 12.90476 & 271.0000 & 399.7281 & 129.5134 \\
\hline NSE 14 & 13.90476 & 292.0000 & 428.6200 & 136.3811 \\
\hline NSE 15 & 14.90476 & 313.0000 & 471.1529 & 144.8923 \\
\hline NSE 16 & 15.90476 & 334.0000 & 499.9281 & 151.7909 \\
\hline
\end{tabular}

SD - standard deviation

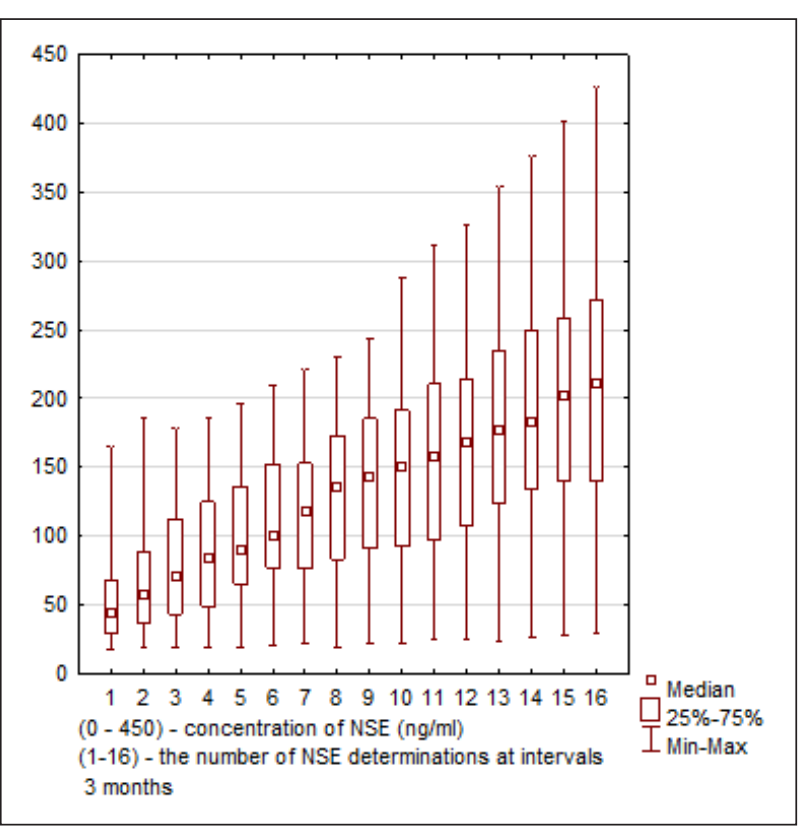

Figure 7. Neuron-specific enolase (NSE) concentrations in patients with stable disease (SD)

involvement $[20,21]$. At the same time, it should be added that NSE is not an ideal marker of neuroendocrine tumours. Its elevated values are also found in small cell lung cancer, neuroblastoma, malignant

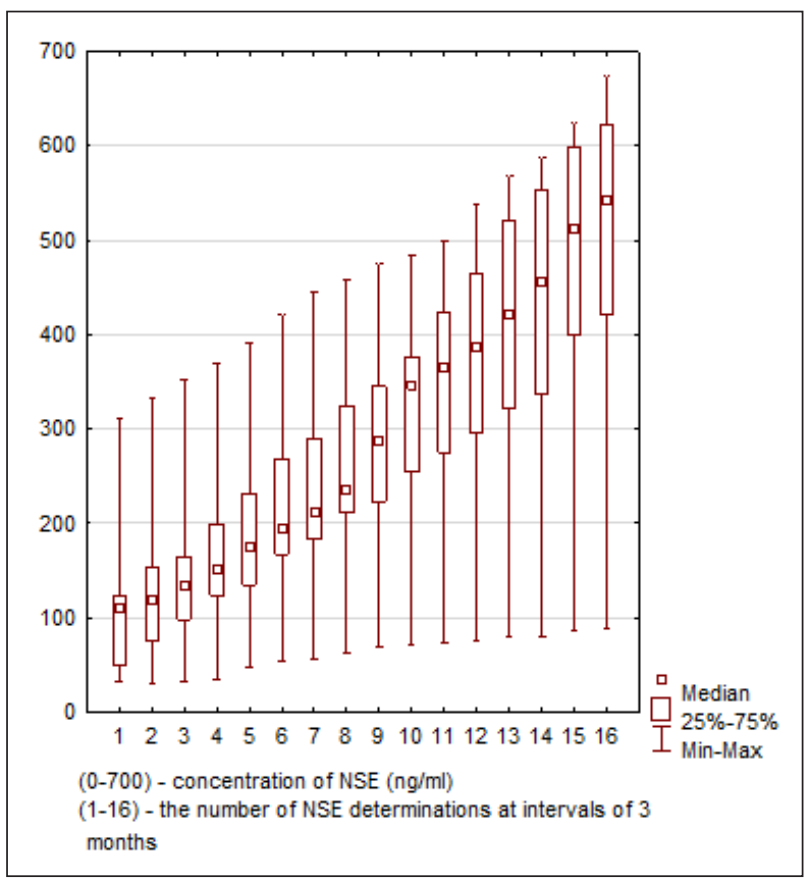

Figure 8. Neuron-specific enolase (NSE) concentrations in patients with progressive disease $(P D)$

melanoma, brain tumours, or inflammation of the central nervous system [22]. Mijones et al. noted that tumour cells with NSE expression are most commonly 


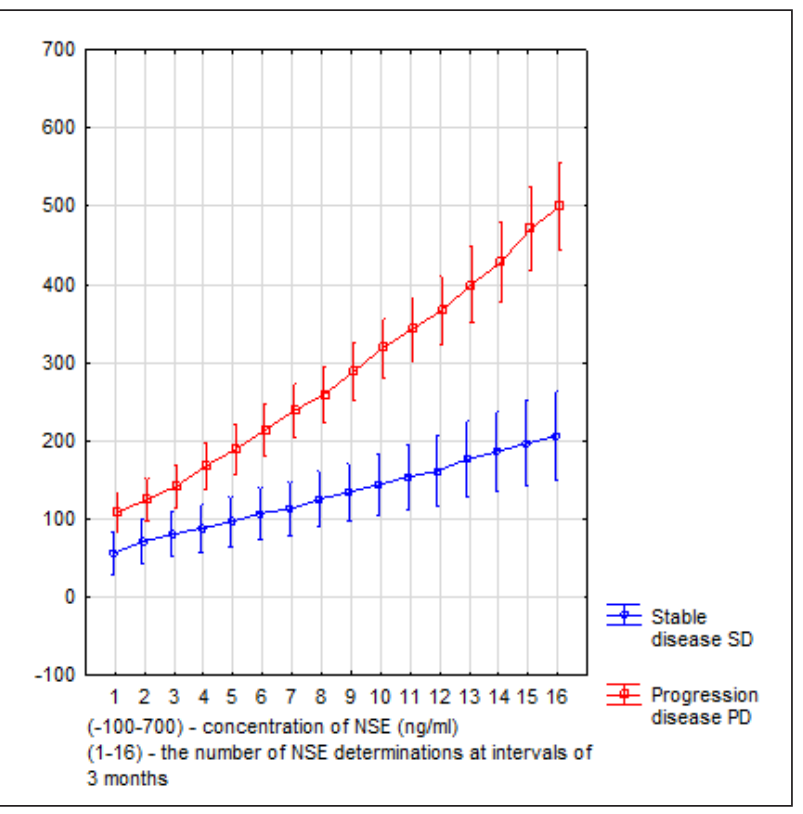

Figure 9. Neuron-specific enolase (NSE) concentrations in patients with stable (SD) and progressive disease (PD)

observed in NETs and renal cell carcinoma. A positive relationship was found between NSE expression and the number of additional neuroendocrine markers expressed in a given tumour, such as $\mathrm{CgA}$, chorionic gonadotropin, and synaptophysin [23]. Similar observations were reported by Bajetta et al., considering that NSE values described in patients with carcinoid tumour are dependent on CgA levels and 5-HIAA excretion. Simultaneous determination of NSE and 5-HIAA showed very high specificity $(100 \%)$ but low sensitivity ( $32.9 \%$ and $35.1 \%$, respectively). Moreover, the results of the above study show that CgA and NSE have the highest sensitivity and most reliable accuracy reflecting the clinical stage of NETs [24]. Completely different findings were described by Manfe et al. evaluating CgA, NSE, and 5-HIAA in small intestinal neuroendocrine tumours with maturity stage G2. The specificity of CgA, NSE, and 5-HIAA was $86 \%, 87 \%$, and $93 \%$, and the sensitivity was $64 \%, 36 \%$, and $35 \%$, respectively. No relationship was found between survival and 5-HIAA excretion and serum NSE levels [25]. In conclusion, it should be emphasized that NSE is one of the key non-specific markers used in the diagnosis and treatment monitoring of neuroendocrine tumours. The sensitivity and specificity of NSE determination is highly applicable in patients with more advanced and aggressive forms of the disease. It is also worth mentioning that the value of NSE determination is significantly higher in correlation with the assessment of other additional markers such as: CgA, 5-HIAA, or synaptophysin.

\section{References}

1. Caplin M, Kvols L. Handbook of neuroendocrine tumours, their current and future management. BioScientisica, Bristol 2006: 103-109.

2. Wick MR, Scheithauer BW, Kovacs K. Neuron-specific enolase in neuroendocrine tumors of the thymus, bronchus, and skin. Am J Clin Pathol. 1983; 79(6): 703-707, doi: 10.1093/ajcp/79.6.703, indexed in Pubmed: 6303108.

3. Giovanella L, La Rosa S, Ceriani L, et al. Chromogranin-A as a serum marker for neuroendocrine tumors: comparison with neuron-specific enolase and correlation with immunohistochemical findings. Int J Biol Markers. 1999; 14(3): 160-166, indexed in Pubmed: 10569138.

4. Kasprzak A, Zabel M, Biczysko W. Selected markers (chromogranin A, neuron-specific enolase, synaptophysin, protein gene product 9.5) in diagnosis and prognosis of neuroendocrine pulmonary tumours. Pol J Pathol. 2007; 58(1): 23-33, indexed in Pubmed: 17585539.

5. Braga F, Ferraro S, Mozzi R, et al. Biological variation of neuroendocrine tumor markers chromogranin A and neuron-specific enolase. Clin Biochem. 2013; 46(1-2): 148-151, doi: 10.1016/j.clinbiochem.2012.09.005, indexed in Pubmed: 23000312

6. Lamberts SW, Hofland LJ, Nobels FR. Neuroendocrine tumor markers. Front Neuroendocrinol. 2001; 22(4): 309-339, doi: 10.1006/frne.2001.0218, indexed in Pubmed: 11587555.

7. Eriksson B, Oberg K, Stridsberg M. Tumor markers in neuroendocrine tumors. Digestion. 2000; 62 Suppl 1: 33-38, doi: 10.1159/000051853, indexed in Pubmed: 10940685.

8. Eberlein-Gonska M, Wiedenmann B, Waldherr R. [Synaptophysin, chromogranin A and neuron-specific enolase as tumor markers in neuroendocrine tumors of the gastrointestinal tract and lung. An immunohistochemical study]. Pathologe. 1989; 10(4): 228-233, indexed in Pubmed: 2549534.

9. Manfé AZ, Norberto L, Marchesini M, et al. Usefulness of chromogranin A, neuron-specific enolase and 5-hydroxyindolacetic acid measurements in patients with malignant carcinoids. In Vivo. 2011; 25(6): 1027-1029, indexed in Pubmed: 22021701.

10. Dittadi R, Gion M. Biological variation of neuroendocrine tumor markers chromogranin A and neuron-specific enolase. Clin Biochem. 2013; 46(12): 1145, doi: 10.1016/j.clinbiochem.2013.04.010, indexed in Pubmed: 23608355.

11. Kos-Kudła B, Blicharz-Dorniak J, Strzelczyk J, et al. Consensus Conference, Polish Network of Neuroendocrine Tumours. [Diagnostic and therapeutic guidelines for gastrointestinal neuroendocrine tumors (recommended by the Polish Network of Neuroendocrine Tumors)]. Endokrynol Pol. 2008; 59(1): 41-56, indexed in Pubmed: 18335400.

12. Kos-Kudła B, Blicharz-Dorniak J, Strzelczyk J, et al. Consensus Conference, Polish Network of Neuroendocrine Tumours. Neuroendocrine neoplasms of the small intestine and the appendix - management guidelines (recommended by the Polish Network of Neuroendocrine Tumours). Endokrynol Pol. 2013; 64(6): 480-493, doi: 10.5603/EP.2013.0029, indexed in Pubmed: 24431119.

13. Landry CS, Cavaness K, Celinski S, et al. Biochemical prognostic indicators for pancreatic neuroendocrine tumors and small bowel neuroendocrine tumors. Gland Surg. 2014; 3(4): 215-218, doi: 10.3978/j. issn.2227-684X.2014.10.01, indexed in Pubmed: 25493250.

14. van Adrichem RCS, Kamp K, Vandamme T, et al. Serum neuron-specific enolase level is an independent predictor of overall survival in patients with gastroenteropancreatic neuroendocrine tumors. Ann Oncol. 2016; 27(4): 746-747, doi: 10.1093/annonc/mdv626, indexed in Pubmed: 26712902

15. Krenning EP, Kwekkeboom DJ, Oei HY, et al. Somatostatin-receptor scintigraphy in gastroenteropancreatic tumors. An overview of European results. Ann NY Acad Sci. 1994; 733: 416-424, doi: 10.1111/j.1749-6632.1994. tb17291.x, indexed in Pubmed: 7978890.

16. Bocchini M, Nicolini F, Severi S, et al. Biomarkers for Pancreatic Neuroendocrine Neoplasms (PanNENs) Management-An Updated Review. Front Oncol. 2020; 10: 831, doi: 10.3389/fonc.2020.00831, indexed in Pubmed: 32537434 .

17. Isgrò MA, Bottoni P, Scatena R. Neuron-Specific Enolase as a Biomarker: Biochemical and Clinical Aspects. Adv Exp Med Biol. 2015; 867: 125-143, doi: 10.1007/978-94-017-7215-0_9, indexed in Pubmed: 26530364.

18. Nobels FR, Kwekkeboom DJ̄, Coopmans W, et al. Chromogranin $A$ as serum marker for neuroendocrine neoplasia: comparison with neuron-specific enolase and the alpha-subunit of glycoprotein hormones. J Clin Endocrinol Metab. 1997; 82(8): 2622-2628, doi: 10.1210/jcem.82.8.4145, indexed in Pubmed: 9253344.

19. Baudin E, Gigliotti A, Ducreux M, et al. Neuron-specific enolase and chromogranin $\mathrm{A}$ as markers of neuroendocrine tumours. $\mathrm{Br}$ J Cancer. 1998; 78(8): 1102-1107, doi: 10.1038/bjc.1998.635, indexed in Pubmed: 9792158.

20. Yao JC, Pavel M, Phan AT, et al. Chromogranin A and neuron-specific enolase as prognostic markers in patients with advanced pNET treated with everolimus. J Clin Endocrinol Metab. 2011; 96(12): 3741-3749, doi: 10.1210/jc.2011-0666, indexed in Pubmed: 21994954. 
21. Korse CM, Taal BG, Vincent A, et al. Choice of tumour markers in patients with neuroendocrine tumours is dependent on the histological grade. A marker study of Chromogranin A, Neuron specific enolase, Progastrin-releasing peptide and cytokeratin fragments. Eur J Cancer. 2012; 48(5): 662-671, doi: 10.1016/j.ejca.2011.08.012, indexed in Pubmed: 21945100.

22. Kaiser E, Kuzmits R, Pregant P, et al. Clinical biochemistry of neuron specific enolase. Clin Chim Acta. 1989; 183(1): 13-31, doi: 10.1016/00098981(89)90268-4, indexed in Pubmed: 2548772.

23. Mjønes P, Sagatun L, Nordrum IS, et al. Neuron-Specific Enolase as an Immunohistochemical Marker Is Better Than Its Reputation. J His- tochem Cytochem. 2017; 65(12): 687-703, doi: 10.1369/0022155417733676, indexed in Pubmed: 28972818.

24. Bajetta E, Ferrari L, Martinetti A, et al. Chromogranin A, neuron specific enolase, carcinoembryonic antigen, and hydroxyindole acetic acid evaluation in patients with neuroendocrine tumors. Cancer 1999; 86(5): 858-865, doi: 10.1002/(sici)1097-0142(19990901)86:5<858::a id-cncr23 > 3.0.co;2-8, indexed in Pubmed: 10463986.

25. Manfé AZ, Norberto L, Marchesini M, et al. Usefulness of chromogranin A, neuron-specific enolase and 5-hydroxyindolacetic acid measurements in patients with malignant carcinoids. In Vivo. 2011; 25(6): 1027-1029, indexed in Pubmed: 22021701. 